\title{
Metas de socialização de pais e mães de diferentes configurações familiares do Rio de Janeiro
}

\section{Parents' socialization goals of different family settings in Rio de J aneiro}

\section{Metas de socialización de padres y madres de diferentes configuraciones familiares de Río de J aneiro}

\author{
Leticia Oliveira da Silva* \\ Pontifícia Universidade Católica do Rio de Janeiro - PUC-Rio, Rio de Janeiro, Rio de \\ Janeiro, Brasil
}

\section{Luciana Fontes Pessôa**}

Pontifícia Universidade Católica do Rio de Janeiro - PUC-Rio, Rio de Janeiro, Rio de Janeiro, Brasil

\begin{abstract}
RESUMO
As metas de socialização têm a sua construção mediada pelas crenças parentais e, por conseguinte, pela cultura, sendo de considerável relevância que sejam investigadas de acordo com os contextos nos quais estão inseridas. Com o objetivo de identificá-las em diferentes tipos de família, participaram do estudo 40 pais e mães residentes na cidade do Rio de Janeiro, com filhos entre sete e 11 anos de idade, de três configurações familiares: 10 casais de famílias nucleares, 10 mães de famílias mononucleares e cinco casais de famílias reconstituídas. Foi utilizado um questionário sociodemográfico para coletar dados como idade, gênero e tipo de família e o Inventário de metas de socialização/desenvolvimento para identificar as trajetórias de socialização. Os resultados apontaram que não houve diferenças significativas entre autonomia e relação na amostra geral $(Z=-0,96 ; p>0,05)$, assim como, não houve diferenças significativas na comparação entre pais e mães nem na dimensão de autonomia $(U=138,50$; $p>0,05)$, nem na dimensão de relação $(U=163,50 ; p>0,05)$. Contudo, famílias nucleares tenderam para metas autônomo-relacionais, enquanto famílias mononucleares e reconstituídas para metas relacionais. Tais resultados sugerem, portanto, a relevância em considerar a pluralidade de arranjos familiares nas investigações das metas de socialização.
\end{abstract}

Palavras-chave: metas de socialização, crenças parentais, diferentes configurações familiares.

\section{ABSTRACT}

Parents' socialization goals have its construction mediated by parental beliefs and therefore by culture, so it's considerably relevant that they be investigated according to the contexts in which they are inserted. In order to identify socialization goals of different family types, 40 parents resident in Rio de Janeiro with children between 7 and 11 years old participated in the 
study. They belonged to three family settings: 10 couples of nuclear families, 10 single mothers of parent families and 5 couples of remarried families. A sociodemographic questionnaire was used to collect data such as age, gender and family type. And the Inventory of socialization/development goals was used to identify the socialization trajectories. Results did not reveal significant differences between autonomy and relatedness in general sample $(Z=-0,96 ; p>0,05)$. As well as did not reveal significant differences in comparison between mothers and fathers, nor in autonomy dimension $(U=138,50 ; p>0,05)$ neither in relatedness dimension $(U=163,50 ; p>0,05)$.. However, nuclear families tended to autonomous relational goals, whereas single patents and remarried families tended for relational goals. Therefore, these results suggest the relevance of considering the plurality of family arrangements in investigation of socialization goals.

Keywords: socialization goals, parental beliefs, different family settings.

\begin{abstract}
RESUMEN
Las metas de socialización tienen su construcción mediada por las creencias parentales $y$, por consiguiente, por la cultura, siendo de considerable relevancia que sean investigadas de acuerdo con los contextos en los que están insertas. Con el objetivo de identificarlas en diferentes tipos de familia, participaron del estudio 40 padres y madres residentes en la ciudad de Río de Janeiro, con hijos entre 7 y 11 años de edad, de tres configuraciones familiares: 10 parejas de familias nucleares, 10 madres de familias mononucleares y 5 parejas de familias reconstituidas. Se utilizó un cuestionario sociodemográfico para recoger datos como edad, género y tipo de familia y el Inventario de metas de socialización/desarrollo para identificar las trayectorias de socialización. Los resultados mostraron que no hubo diferencias significativas entre autonomía y relación en la muestra general $(Z=-0,96 ; p>0,05)$. Así como, no hubo diferencias significativas en la comparación entre padres y madres ni en la dimensión de autonomía $(U=138,50 ; p>0,05)$ ni en la dimensión de relación $(U=163,50 ; p>0,05)$. Sin embargo, las familias nucleares se inclinaron a metas autónomarelacionales, mientras las familias mononucleares y reconstituidas a metas relacionales. Por lo tanto, tales resultados sugieren la relevancia de considerar la pluralidad de arreglos familiares en las investigaciones de las metas de socialización.
\end{abstract}

Palabras clave: metas de socialización, creencias parentales, diferentes configuraciones familiares.

\title{
1 I ntrodução
}

O ser humano é extremamente dependente em seus primeiros anos de vida. Nenhuma outra espécie é tão vulnerável ao nascer como o Homo sapiens. Assim, a família humana, mais especificamente a parentalidade, possui a função central de proporcionar às crianças um contexto para o desenvolvimento de competências apropriadas à ecologia local, onde se inserem essas famílias (Geary \& Flinn, 2001). Nesse sentido, o exercício da parentalidade tende a ser orientado por um conjunto de crenças, denominadas por Harkness e Super (2006) como etnoteorias parentais. Para esses autores, as etnoteorias parentais consistem em modelos culturais que os pais detêm sobre 
filhos, famílias, e eles próprios como pais. Tais modelos seriam, portanto, produto de um sistema interativo entre ambiente $\mathrm{e}$ indivíduo (Keller \& Greenfield, 2000).

De acordo com Harkness e Super (2006), as etnoteorias parentais não podem ser pensadas fora do que conceituam como "nicho do desenvolvimento" (Harkness \& Super, 1993), quadro teórico que compreende o desenvolvimento infantil através de modelos culturais e que estariam divididos em três componentes ou subsistemas: a) configurações físicas e sociais em que a criança vive; b) costumes culturalmente construídos sobre os cuidados infantis e a parentalidade e; c) psicologia dos cuidadores - subsistema que inclui as crenças e as expectativas dos pais e/ou dos demais cuidadores.

As crenças parentais são construídas ao longo da vida, na medida em que, durante o processo de socialização, meninos e meninas são orientados sobre os papéis que podem vir a desempenhar quando crescerem, dentre eles, o de mãe/pai (Seidl-de-Moura, 2003). Contudo, isso não significa que tais crenças não podem ser alteradas quando, de fato, essas crianças tornarem-se adultas e passarem a exercer o papel parental.

A esse respeito, Ligthfoot e Valsiner (1992) afirmam que as crenças parentais funcionam como organizadores mentais para as ações, mas não correspondem, necessariamente, às práticas parentais. Conforme os autores, isso ocorre porque, além das crenças serem construídas nas relações sociais e receberem influência do contexto, elas contam com a participação de um sujeito que também atua nessa construção com suas idiossincrasias. Portanto, de acordo com as crenças parentais, pais, mães e demais cuidadores podem privilegiar diferentes metas de socialização em suas práticas parentais, metas e práticas estas que serão essenciais para o desenvolvimento infantil.

\section{Metas de Socialização}

As metas de socialização tendem a ser apoiadas por diferentes trajetórias de socialização. Tais trajetórias têm sido classificadas em três diferentes modelos. O primeiro modelo, denominado independente ou autônomo, dá ênfase às metas pessoais, priorizando as necessidades e os direitos do indivíduo. Nesse modelo, as dimensões da separação e da autonomia - características de sociedades urbanas pós-industriais - predominam (Kağitçibaşi, 2007; Keller, Borke, Yovsi, Lohaus, \& Jensen, 2005).

O segundo deles, o modelo interdependente ou relacional, enfatiza as metas grupais, assim como os papéis sociais, os deveres e as obrigações. Prevalecem, assim, as dimensões da heteronomia e da relação, que caracterizam ambientes rurais baseados em economia de subsistência (Kagitçibasi, 2007; Keller et al., 2005). 
Por fim, o modelo autônomo-relacional abrange características de ambos os modelos anteriores, englobando tanto a autonomia quanto a relação, o que é característico de famílias de classe média urbana em sociedades tradicionalmente interdependentes (Kagitçibasi, 2005, 2007). De acordo com Kağitçibaşi (2012), para o atual mundo globalizado, esse modelo pode ser entendido como uma meta de desenvolvimento sadia, na medida em que, consiste num modelo de adaptação que atende tanto às demandas contemporâneas quanto às demandas da espécie, unindo a atuação autônoma do indivíduo à valorização do ato de se relacionar e cooperar com os demais.

Pode-se entender, portanto, que autonomia e relação se manifestam de diferentes formas de acordo com as demandas contextuais de cada ambiente cultural. Keller (2012) conceitua três diferentes ambientes em que essas dimensões possuem significados diversos: protótipos de famílias ocidentais, urbanas e de classe média que tendem para trajetória autônoma; protótipos de famílias rurais, com agricultura de subsistência que tendem para trajetória relacional; e um meio híbrido de famílias de classe média urbana de ambientes não-ocidentais que apresentam tanto características autônomas quanto relacionais, tendendo para trajetória autônomo-relacional.

Pesquisas na área têm demonstrado as diferentes valorizações das metas de socialização por pais residentes em contextos culturais diversos (Jaramillo, Pérez, \& González, 2013; Vieira, Martins, \& Lordelo, 2013). Os resultados de tais pesquisas sugerem que mães que vivem em contextos mais independentes, tendem a priorizar metas relacionadas ao desenvolvimento da autonomia de seus filhos. Por outro lado, mães residentes em contextos mais interdependentes, valorizam o bom comportamento da criança e sua adequação às expectativas sociais.

Um estudo colombiano, realizado por Jaramillo et al. (2013) com 163 mães residentes em contextos rural e urbano, investigou as correlações entre metas de socialização, zona habitacional, nível de escolaridade e idade da mãe. Os resultados indicaram que mães de zonas urbanas, com menor idade e de maior nível de escolaridade tenderam para metas de socialização autônomas, enquanto mães de mais idade e de menor nível acadêmico priorizaram metas relacionais.

Vieira et al. (2013) obtiveram resultados semelhantes em contexto brasileiro. Ao compararem as crenças de mães da capital e de uma cidade pequena, os autores observaram uma diferença significativa em relação às variáveis sociodemográficas (idade e nível de escolaridade das mães, renda familiar etc.) dos dois contextos. Além disso, na capital, tanto as práticas quanto as metas de socialização relacionadas à autonomia foram mais valorizadas do que na pequena cidade. Por outro lado, as mães que vivem na cidade pequena valorizaram mais a dimensão relacional, tanto para práticas quanto 
para metas de socialização. Contudo, as médias de autonomia e relação forma muito próximas em ambos os contextos, indicando uma avaliação média de práticas e objetivos relacionais e autônomos. Resultados próximos foram encontrados por Westphal, Vieira, Vieira, e Prado (2010), ao entrevistarem 150 mães de três contextos com diferentes índices de urbanização em Santa Catarina: capital, interior leste e interior oeste. Os resultados apontaram para a presença do modelo autônomo-relacional nos três contextos. Contudo, a dimensão alocêntrica e as metas relacionais se correlacionaram positivamente, indicando que quanto mais próximas da família as mães relataram ser, mais elas tendiam a estabelecer metas de socialização relacionais para os filhos.

Borges e Salomão (2015), por outro lado, se dedicaram ao estudo do contexto não urbano. Com o objetivo de identificar as concepções maternas sobre o desenvolvimento infantil e suas metas de socialização, as autoras entrevistaram 30 mães residentes em área não urbana de Petrolina (PE), com filhos entre seis e 36 meses de idade. Os resultados revelaram concepções maternas positivas em relação ao desenvolvimento de seus filhos, à valorização de cuidados com a saúde, afetividade, educação e socialização da criança. Além disso, valorizaram tanto 0 autoaperfeiçoamento quanto as expectativas sociais, apresentando metas orientadas para o modelo autônomo-relacional. Tais resultados são explicados pelas autoras em função da proximidade com o contexto urbano, do nível de escolaridade materno (geralmente ensino médio completo) e do reduzido número de filhos.

Em estudo nacional, Seidl-de-Moura et al. (2013) observaram resultados análogos. Participaram da pesquisa 94 mães brasileiras, com filho de 17 a 22 meses de idade, a fim de investigar como descrevem seus filhos e a presença de autonomia e relação em suas descrições. Os resultados indicaram que os adjetivos mais utilizados foram "inteligente" e "ativo", associados à autonomia, e "amoroso" e "carinhoso", associados à relação. Portanto, não foram encontradas diferenças significativas entre os adjetivos descritores de independência e os adjetivos descritores de interdependência, o que de acordo com os autores, sugere a tendência para um modelo autônomo-relacional.

Como pode ser constatado nos estudos descritos, atualmente, parece haver uma certa homogeneidade cultural no contexto brasileiro, provavelmente em função da globalização, que aponta o modelo autônomo-relacional como predominante. No entanto, tais estudos são normalmente realizados com mães de crianças pequenas.

Nesse sentido, com o objetivo de ampliar a investigação de pesquisas brasileiras sobre trajetórias de socialização, Seidl-de-Moura et al. (2017) realizaram dois estudos: o primeiro, com avós(ôs), pais, mães e filhos, com idade de 16 a 25 anos, e o segundo, com grupos de 
cuidadoras de crianças até um ano de idade, com diferentes níveis de escolaridade. Os resultados apontaram que, apesar de mães criadas na cidade do Rio de Janeiro e pais com maior nível de escolaridade apresentarem médias maiores de autonomia, o modelo autônomorelacional preponderou para todos os participantes, de ambos estudos.

Outros estudos brasileiros têm se esforçado para a inclusão da figura paterna em relação à investigação das crenças parentais. Buscando analisar as metas de socialização de pais e mães em relação aos seus filhos, Lins, Salomão, Borges, Lins, e Carneiro (2015) realizaram um estudo com 18 homens e 18 mulheres residentes em João Pessoa $(\mathrm{PB})$, casados entre si e que tinham apenas um filho. Os resultados mostraram que as metas mais valorizadas tanto por pais quanto por mães foram o respeito aos outros, honestidade, êxito escolar, êxito pessoal e afetividade. Ao comparar as metas de socialização entre pais e mães, os resultados demonstraram mais semelhanças do que diferenças. Embora metas relacionais tenham apresentado um percentual elevado, pais e mães tenderam para um modelo autônomo-relacional.

De forma semelhante, Fortkamp, Vieira, e Faraco (2015) investigaram diferenças e semelhanças relacionadas às crenças parentais e metas de socialização, assim como fatores que estão associados às práticas dos cuidadores. Participaram da pesquisa 48 pais e mães, residentes em Florianópolis (SC), com filhos de quatro a seis anos de idade e de dois contextos com diferentes níveis de escolaridade. Os resultados apontaram que as crenças e práticas parentais, em ambos os contextos, indicam o modelo autônomorelacional. Contudo, os pais com maior escolaridade privilegiaram atributos de autonomia, como independência psicológica e assertividade, enquanto os pais de menor escolaridade enfatizaram a importância de saber fazer escolhas e estudar para obter melhores condições de vida, reforçando a obediência e o respeito às regras.

Pesquisas realizadas no Rio de Janeiro também têm se debruçado sobre o estudo das trajetórias de socialização a partir do viés de diferentes cuidadores. Com o objetivo de explorar o que cuidadores pensam sobre a expressão emocional das crianças e as possíveis relações com as metas de socialização priorizadas, Mendes e Pessôa (2013) entrevistaram 20 duplas, residentes na cidade do Rio de Janeiro, compostas por mães e outros cuidadores que compartilhavam os cuidados de seu filho de até um ano de idade. Os resultados apontaram uma valorização, por parte das mães, para que seus filhos aprendam a controlar suas emoções antes dos três anos de idade, o que consiste numa característica associada à interdependência. Por outro lado, tais mães também demonstraram uma maior valorização do sorriso enquanto expressão de emoção em 
detrimento a uma forma de expressar simpatia, o que pode estar mais relacionado à autonomia.

De maneira similar, em estudo com 60 duplas da cidade do Rio de Janeiro, formadas por mães de filhos com até um ano de idade e avós, babás ou educadores de creche, Pessôa, Seidl-de-Moura, Ramos e Mendes (2016) investigaram os sistemas parentais através de fotos que representavam cada um desses sistemas. Os sistemas mais valorizados pelas participantes foram os de contato corporal e troca face a face, o que, de acordo com as autoras, aponta para um modelo autônomo-relacional, uma vez que o contato corporal é predominante em contextos onde a relação é valorizada e a troca face a face é prevalente em contextos mais autônomos.

Interessados nas concepções maternas e paternas, Bandeira, Seidlde-Moura e Vieira (2009) encontraram resultados semelhantes. A fim de investigar as metas de socialização privilegiadas por mães e pais, foram entrevistados trinta casais do Rio de Janeiro, com filho único de até três anos. Os resultados do estudo indicaram que pais e mães valorizavam a autonomia, mas também a interdependência, o que sugere que ambos possuíam tendência de socialização para autonomia-relacional.

Como visto até aqui, parece, portanto, haver uma tendência atual para o modelo autônomo-relacional no contexto brasileiro, assim como na cidade do Rio de Janeiro, independentemente do gênero do cuidador, o que está de acordo com a teoria, uma vez que, em famílias de classe média urbana de sociedades tradicionalmente interdependentes, o modelo autônomo-relacional tende a predominar (Kagitçibasi, 2005, 2007).

No entanto, também pode-se perceber que diversos fatores podem contribuir significativamente para a prevalência de metas autônomas e/ou relacionais. Além de aspectos da cultura maior, características sociodemográficas, como nível de escolaridade, e o tipo de contexto (urbano ou rural) parecem ter forte influência no conjunto de crenças e práticas dos cuidadores, inclusive nas metas de socialização que priorizam para educação de seus filhos.

Grande parte das pesquisas realizadas na área contam somente com a participação de pais e mães de famílias nucleares com filhos em idade pré-escolar. Diante da possibilidade de influência de características sociodemográficas, parece relevante questionar se a tendência autônomo-relacional se mantém na medida em que criança torna-se mais velha e se também se estende a outras configurações familiares. Nesse sentido, torna-se importante entender um pouco mais sobre a construção histórico-social da família, o contexto cultural em que ocorreram suas transformações, assim como suas diferentes configurações. 


\section{As Diferentes Configurações Familiares e Sua Construção Histórico-Cultural}

O termo "família" é derivado do latim famulus, cujo significado refere-se ao grupo de dependentes e servos de um senhor (Wagner, 2002). De acordo com Pinheiro e Biasoli-Alves (2008), tal termo foi criado na Roma Antiga para denominar um grupo social introduzido na agricultura e na escravidão legalizada. Nesse período, predominava uma estrutura familiar patriarcal, devido à submissão desses indivíduos à autoridade de um mesmo chefe. No entanto, a partir da Idade Média, novas configurações familiares foram formadas através das uniões matrimoniais - que selavam os acordos familiares. De acordo com Passos (2015), a família passou por três parâmetros históricos. Até o século XIX, o casamento era definido/arranjado pelos pais, com o objetivo de preservar o patrimônio da família. Além disso, as dimensões afetiva e sexual, raramente, se faziam presentes e todos os membros da família eram submetidos à inquestionável autoridade patriarcal (Passos, 2015).

A partir da Revolução Industrial, um segundo momento (final do século XIX até meados do século XX) é inaugurado. Os jovens, ao se deslocarem para as cidades em busca de trabalho em fábricas, adquirem liberdade para escolher seus parceiros. As relações conjugais passam, assim, a ser pautadas no afeto e na sexualidade. As relações pais-filhos também se tornam mais íntimas, porém, com o monitoramento dos filhos pela autoridade paterna e pela educação materna repressiva (Passos, 2015).

Após a Segunda Guerra Mundial, surgem alguns movimentos de combate à dominação (feminismo, políticas de sexualidade etc) que influenciam no questionamento do poder paterno. Desse modo, na segunda metade do século $X X$, emerge o terceiro e atual momento da família. A força política paterna entra em um processo de desconstrução: o poder não está mais exclusivamente nas mãos do pai, mas circula no interior da família, o que gera novos posicionamentos tanto das figuras de autoridade (pai e mãe) quanto dos que a ela se submetem (Passos, 2015).

Diante do cenário apresentado, é possível notar o entrelaçamento entre os acontecimentos sociohistóricos e as estruturas familiares, tão plurais e complexas na sociedade contemporânea. Nesse sentido, Pinheiro e Biasoli-Alves (2008) alertam para a dificuldade de se conceituar a família na atualidade, uma vez que se corre o risco de excluir formas diferenciadas de constituição familiar.

Kaslow (2002) classifica oito tipos de composições familiares: a) família nuclear; b) família extensa (inclui três ou quatro gerações); c) família adotiva; d) casal; e) família monoparental; f) família homoafetiva/homoparental; g) família reconstituída; h) família sem laços legais. No que concerne ao presente estudo, faz-se necessária a 
descrição de três tipos diferentes de família: nuclear, mononuclear e reconstituída.

De acordo com o autor, a família nuclear inclui duas gerações com filhos biológicos, ou seja, ela é composta por um homem e uma mulher que coabitam e possuem ao menos um filho. Já as famílias monoparentais ou mononucleares, são formadas por um dos progenitores, o pai ou a mãe, e por seu(s) filho(s) ainda não adultos. Dados do último censo, realizado pelo Instituto Brasileiro de Geografia e Estatística [IBGE] (2010), apontam que a principal configuração familiar brasileira ainda é casal com filhos, ou seja, nuclear $(47,3 \%)$. Todavia, o segundo arranjo familiar mais presente no Brasil é o mononuclear feminino, composto por mulher e filhos $(17,4 \%)$, seguido por casal sem filhos $(17,1 \%)$, e outros tipos $(6,2 \%)$.

Embora a monoparentalidade possa ser percebida como oportunidade de crescimento pessoal, existe a possibilidade desses genitores se sentirem sobrecarregados por serem os únicos responsáveis pelos cuidados demandados pela criança, podendo, por vezes, não dar conta das necessidades que apresentam (Mota \& Matos, 2011). Enquanto o recasamento, pode implicar em certa resistência da criança em relação ao novo cônjuge do progenitor, fazendo com que os filhos de famílias reconstituídas apresentem resultados desenvolvimentais mais baixos em relação às crianças de famílias nucleares (Jensen \& Shafer, 2013).

Ainda são raros os estudos nacionais que investigam as implicações que as diferentes configurações familiares podem trazer para 0 exercício da parentalidade e para o desenvolvimento infantil. Dentre eles está o estudo de Leme, Del Prette e Coimbra (2013), que analisou as habilidades sociais e as percepções sobre práticas educativas maternas de 454 adolescentes de famílias nucleares, mononucleares e reconstituídas. Os resultados apontaram não haver influência da configuração familiar nas práticas educativas maternas. No entanto, adolescentes de famílias reconstituídas apresentaram mais habilidades sociais de abordagem afetiva em relação aos adolescentes de famílias nucleares e mononucleares.

Igualmente interessadas em possíveis relações entre configurações familiares e parentalidade, Pessôa, Ramos, Seidl-de-Moura e Silva (2018) investigaram as metas de socialização da obediência infantil de famílias nucleares, mononucleares e reconstituídas. Embora os resultados não tenham apontado diferenças significativas na valorização das metas de socialização da obediência entre os tipos de família, notou-se que em famílias reconstituídas houve uma maior valorização da obediência em pais e mães de meninas em comparação a pais e mães de meninos. Além disso, em famílias monoparentais observou-se que quanto mais novos são os filhos, mais a obediência é valorizada. 
Diante da escassez de estudos nacionais que abordem as metas de socialização em diferentes configurações familiares, a presente pesquisa tem por objetivo identificar a predominância de metas autônomas e/ou relacionaisem diferentes tipos de família. Para tanto, são levantadas as seguintes questões: Qual das dimensões, autonomia e/ou relação, é predominante na amostra geral? Será que famílias nucleares tendem para autonomia-relacional, como sugerem estudos anteriores?E famílias reconstituídas? Será que, por se contituírem a partir da união de duas famílias, enfatizam os papéis sociais, privilegiandometas relacionais? E as famílias mononucleares? Será que, diante do fato dessas mães criarem seus filhos sozinhas, tendem a priorizar metas autônomas? E em relação ao genêro dos cuidadores? Será que pais e mães privilegiam metas autônomosrelacionais como indica a literatura?

\section{Método}

\subsection{Participantes}

Partiparam da pesquisa pais e mães de 25 famílias da cidade do Rio de Janeiro (10 nucleares, 10 monoparentais e cincoreconstituídas), com pelo menos um filho entre setee 11 anos de idade $(M=8,60$; $\mathrm{DP}=1,30$ ). Responderam, portanto, aos questionários um total de 40 sujeitos, sendo eles: 10 pais e 10 mães das famílias nucleares, cincopais e cincomães das famílias reconstituídas e 10 mães das famílias monoparentais. Em relação às crianças, 20 eram meninas e 20 eram meninos.

\subsection{I nstrumentos}

- Questionário sociodemográfico: coleta de informações pertinentes àpesquisa, tais como idade, gênero, situação conjugal dos cuidadores etc.

- Inventário de metas de socialização/desenvolvimento: inventário de comparação de metas de socialização (Kelleret al., 2006; adaptada por Seidl-de-Moura et al., 2008): escala composta por 10 itens que indicam opiniões sobre metas que os pais vão tentar alcançar no desenvolvimento de seus filhos. Pede-se que indique se concorda ou não com cada item, em uma escala de 1 (se não concorda nem um pouco) a 5 (se concorda totalmente). A análise fatorial de componentes principais produziu duas dimensões: de metas de autonomia (cinco itens), como por exemplo, "desenvolver competitividade" e de metas relacionais (cinco itens), tais como "obedecer aos mais velhos". As subescalas das duas dimensões apresentaram índices adequados de fidedignidade (Alfas de 
Crombach de 0,93 para a subescala de metas de autonomia e 0,89 para a de metas relacionais). As duas medidas não se correlacionaram significativamente.

\subsection{Procedimentos Éticos}

O projeto foi aprovado pelo Comitê de Ética da PUC-Rio (2017-22). Todos os participantes receberam informaçãooral e escrita sobre os objetivos da pesquisa, responsabilidade, método empregado e direito a se recusar a participar a qualquer momento durante a realização da pesquisa.

A inclusão no grupo de participantes foi condicionada à assinatura do Termo de Conscentimento Livre e Esclarecido. Cada um dos participantes foi identificado por um código nas folhas dos instrumentos e seus dados pessoais constavam somente na ficha sociodemográfica, tendo, assim, suas identidades preservadas.

\subsection{Procedimentos de Coleta de Dados}

O contato com as famílias foi realizado através de indicação dos mebros do grupo de pesquisa Desenvolvimento, Biologia e Cultura $(\mathrm{DBC})$. No primeiro contato com os cuidadores, 0 pesquisador solicitava sua cooperação, explicando os objetivos e método da pesquisa numa linguagem simples e clara. Havendo concordância na participação, o pesquisador pedia a assinatura do Termo de Consentimento Livre e Esclarecido e procedia à aplicação do questionário sociodemográfico e da escala.

\subsection{Procedimentos de Análise de Dados}

As metas de socialização, que envolvem as dimensões de autonomia e relação, foram analisadas através de testes não paramétricos, uma vez que o teste de normalidade (Kolmogorov-Smirnov) indicou distribuição aproximadamente normal da dimensão de autonomia, mas não da dimensão de relação (teste $K S=p<0,05$ ). Dessa forma, além de estatísticas descritivas (média e desvio padrão), foram realizados o teste Wilcoxon para verificar a existência de diferença significativa entre autonomia e relação na amostra como um todo e o teste $U$ de Mann-Whitney para testar diferenças nas dimensões entre pais e mães. Em relação às diferenças de acordo com o tipo de família, as dimensões foram analisadas somente de forma descritiva devido ao baixo tamanho amostral. 


\section{Resultados e Discussão}

Os resultados indicaram que a média das metas de relação na amostra geral $(M=20,15 ; D P=15,24)$ foi mais elevada do que a média das metas de autonomia $(M=17,47 ; \mathrm{DP}=3,78)$. No entanto, o teste Wilcoxon revelou não haver diferença significativa entre essas dimensões $(Z=-0,96 ; p>0,05)$.

De forma semelhante, quando as dimensões foram comparadas de acordo com o gênero do cuidador (ver Figura 1), mães apresentaram médias de metas autônomas mais altas $(M=18,04 ; D P=3,65)$ do que os pais $(M=16,53 ; D P=3,94)$. Enquanto em relação às metas relacionais, pais obtiveram médias superiores $(M=23,40 ; D P=24,69)$ quando comparadas às mães $(M=18,20 ; D P=3,33)$. Entretanto, 0 teste $U$ de Mann-Whitney indicou não haver diferenças significativas na comparação entre pais e mães nem na dimensão de autonomia $(U=138,50 ; p>0,05)$ nem na dimensão de relação $(U=163,50$; $p>0,05)$. Ademais, entende-se que o tamanho amostral e a atribuição de valores extremos por pais à dimensão de relação podem ter influenciado o resultado do teste de significância.

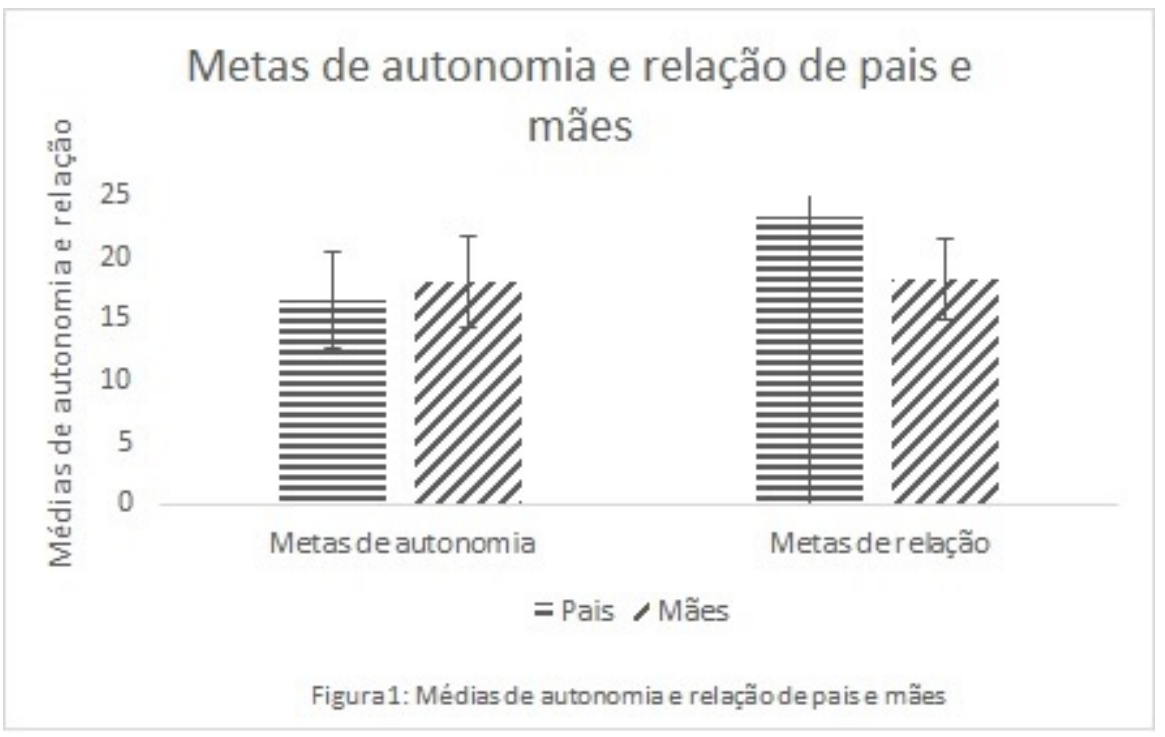

Quando comparadas as metas de autonomia e relação nos diferentes tipos de família, como pode ser observado na Figura 2, os cuidadores de famílias nucleares valorizaram da mesma forma as dimensões de autonomia $(M=17,80 ; D P=3,97)$ e relação $(M=17,80 ; D P=3,16)$. No entanto, pais e mães de famílias mononucleares e reconstituídas valorizaram mais as metas de relação $(M=19,10 ; D P=1,60$ e $M=25,90 ; D P=30,53)$ quando comparadas às metas de autonomia $(M=18,00 ; D P=1,70$ e $M=16,30 ; D P=4,88)$. Contudo, não foi possível realizar um teste de significância entre essas amostras devido ao seu tamanho. Além disso, a amostra de famílias reconstituídas 
apresentou um desvio bastante elevado na dimensão de relação, o que pode apontar para valores extremos atribuídos às metas dessa dimensão.

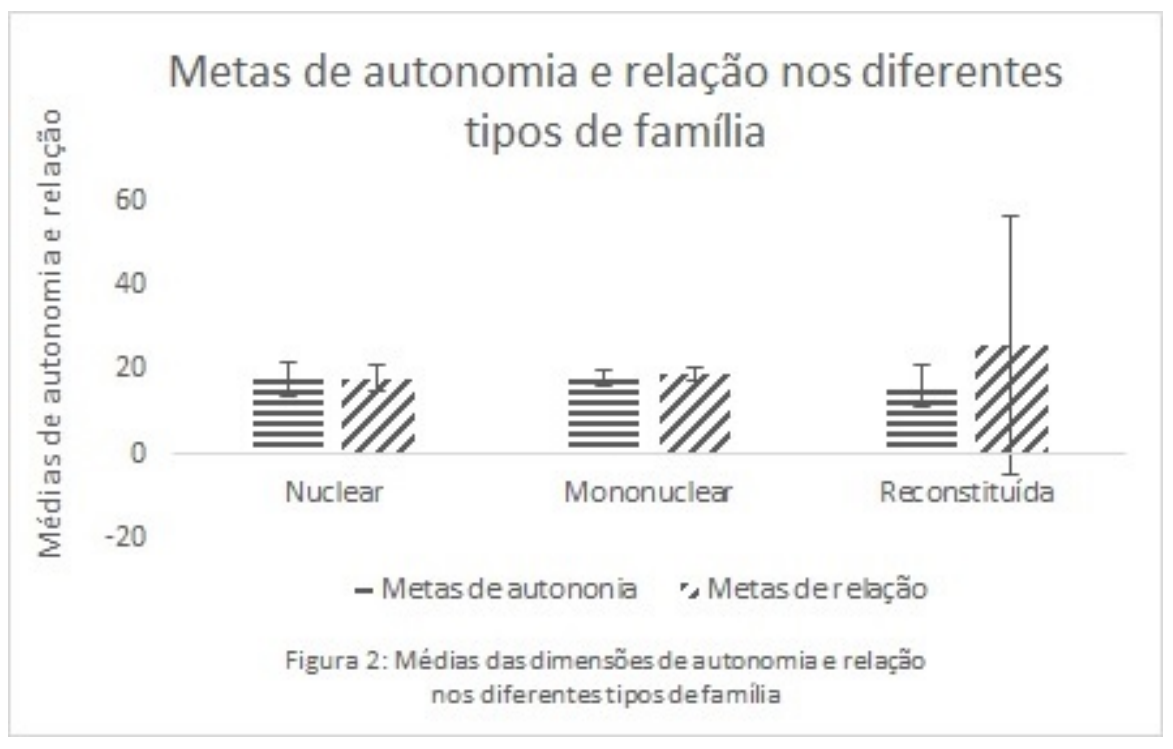

Portanto, os resultados desse estudo indicaram não haver diferença significativa entre as dimensões de autonomia e relação dos participantes em geral, o que caracteriza a amostra com o modelo autônomo-relacional, típico em famílias de classe média urbana em sociedades tradicionalmente interdependentes (Kagitçibasi, 2005, 2007). Tal resultado está em consonância com estudos anteriores nos quais cuidadores residentes em capitais valorizaram igualmente as dimensões de autonomia e relação em suas metas de socialização (Mendes \& Pessôa, 2013; Pessôa et al., 2016; Seidl-de-Moura et al., 2013; Seidl-de-Moura et al., 2017).

Além disso, apesar de pais apresentarem maiores médias na dimensão de autonomia e de mães apresentarem maiores médias na dimensão de relação, não foram encontradas diferenças significativas na comparação entre pais e mães em nenhuma das dimensões. Tal resultado também corrobora com a literatura, que indica que pais e mães valorizam a autonomia, mas também a relação, sugerindo que ambos possuem tendência de socialização para autonomiarelacional(Bandeira et al., 2009; Fortkamp et al., 2015; Lins et al., 2015; Seidl-de-Moura et al., 2017). Contudo, o baixo nível de significância entre as diferenças também pode ter sido influenciado pela presença de valores extremos e pelo reduzido tamanho amostral.

Em relação aos tipos de famílias, pais e mães de famílias nucleares apresentaram médias idênticas nas dimensões de autonomia e relação, também apontando para um modelo autônomo-relacional, como sugerido em estudos anteriores (Bandeira et al., 2009; Lins et 
al., 2015). No entanto, embora não tenha sido possível realizar um teste de significância, cuidadores de famílias monoparentais e reconstituídas apresentaram médias mais elevadas em metas relacionais.

De acordo com o estudo de Westphal et al. (2010) sobre metas de socialização e alocentrismo familiar, há uma correlação positiva entre metas relacionais e a dimensão alocêntrica, o que indica que quanto mais próximas da família as mães relatam ser, mais elas tendem a estabelecer metas de socialização relacionais para os filhos. Nesse sentido, é possível que em famílias reconstituídas, nas quais há um número maior de pessoas, devido à junção de membros da família anterior com membros da atual família, a proximidade familiar seja mais evidente, o que pode explicar a prevalência de metas relacionais nesse tipo de família. No entanto, deve-se considerar o elevado desvio padrão das metas de relação nessa configuração familiar, o que pode indicar a influência de valores extremos na média obtida.

Em relação às mães de famílias mononucleares, ainda que não haja um respaldo teórico a respeito, a hipótese formulada é a de que, o fato de por vezes o(s) filho(s) serem suas únicas companhias no ambiente domiciliar, a dimensão relacional ganhe um peso maior, na medida em que a autonomia pode levar à independência da criança em relação a essas mães.

\section{Considerações Finais}

As crenças ou etnoteorias parentais são construídas nas relações sociais sendo fortemente influenciadas pelo contexto. Sendo assim, funcionam como organizadores mentais para as ações, mas não correspondem, necessariamente, às práticas parentais, uma vez que a sua construção conta com um sujeito que é atuante com suas idiossincrasias (Ligthfoot \& Valsiner, 1992).

No que diz respeito ao estudo das trajetórias de socialização, são essas crenças que tendem a orientar as metas priorizadas por pais e mães no desenvolvimento de seus filhos. Nesse sentido, acredita-se que o presente estudo possa ter contribuído de forma significativa para o estudo das crenças parentais e das metas de socialização, na medida em que apontou a predominância do modelo autônomorelacional nos participantes em geral e nas amostras de pais e de mães, corroborando com estudos anteriores (Bandeira et al., 2009; Fortkamp et al., 2015; Lins et al., 2015; Seidl-de-Moura et al., 2017).

Além disso, em relação aos tipos de família, apesar de famílias nucleares terem apresentado trajetória autônomo-relacional e famílias reconstituídas, trajetória relacional, diferentemente do esperado, famílias mononucleares também tenderam para trajetória 
relacional. No caso de famílias reconstituídas, tal resultado parece estar associado a um possível alocentrismo familiar, enquanto que em famílias mononucleares, ao fato dos filhos serem, por vezes, a única companhia de suas mães, fazendo com que a dimensão relacional tenha um maior destaque.

Contudo, há a possibilidade de que valores extremos apresentados pela amostra tenham influenciado de alguma forma esses resultados, uma vez que não puderam ser excluídos devido ao baixo tamanho amostral. Além do mais, apenas um instrumento foi utilizado para a mensuração das trajetórias de socialização, o que pode ter revelado uma avaliação incompleta do fenômeno. Por fim, somente três configurações familiares foram investigadas e algumas variáveis importantes para o estudo das metas de socialização, como nível de escolaridade e nível socioeconômico (Borges \& Salomão, 2015; Fortkamp et al., 2015; Jaramillo et al., 2013; Vieira et al., 2013), não foram consideradas.

Nesse sentido, torna-se necessário que estudos posteriores avaliem uma maior amostragem para viabilizar testes de significância que não puderam ser realizados nesse estudo. Ademais, para pesquisas futuras, sugere-se que mais de um instrumento seja utilizado para avaliação do fenômeno, que outras configurações familiares sejam investigadas e que outras variáveis sociodemográficas sejam consideradas.

Entretanto, acredita-se que o presente trabalho pode ser um achado científico, na medida em que corrobora com resultados de estudos anteriores e lança luz sobre novos aspectos ainda não investigados pela literatura. Desse modo, suas contribuições vão em direção de colaboração com a Psicologia do Desenvolvimento e com o estudo das metas de socialização, possibilitando a abertura de caminho para novas investigações.

\section{Referências}

Bandeira, T. T. A., Seidl-de-Moura, M. L., \& Vieira, M. L. (2009). Metas de socialização de pais e mães para seus filhos. Revista Brasileira de Crescimento e Desenvolvimento Humano, 19(3), 445-456.

Borges, L. C., \& Salomão, N. M. R. (2015). Concepções de desenvolvimento infantil e metas de socialização maternas em contexto não urbano. Estudos de Psicologia, 20(2), 114-125.

Fortkamp, E. H. T, Vieira, M. L., \& Faraco, A. M. X. (2015). Crenças e Metas de Socialização de Pais de dois Contextos Urbanos Brasileiros: Uma Análise do Modelo de Orientação de Self (Tese de doutorado). Universidade Federal de Santa Catarina, 
Trindade, Florianópolis. Retirado de https://repositorio.ufsc.br/handle/123456789/156521

Geary, D. C., \& Flinn, M. V. (2001). Evolution of human parental behavior and the human family. Parenting: Science and Practice, 1(2), 5-61.

Harkness, S., \& Super, C. M. (1993). The developmental niche: Implications for children's literacy development. In L. Eldering \& P. Lesemen (Eds.), Early intervention and culture: Preparation for literacy (pp. 115-132). Paris: UNESCO.

Harkness, S., \& Super, C. M. (2006). Themes and variations: parental ethnotheories in western cultures. In K. Rubin (Ed.), Parental beliefs, parenting, and child development in cross-cultural perspective (pp. 61-79). New York: Psychology Press.

Instituto Brasileiro de Geografia e Estatística [IBGE]. (2010). Estatísticas do registro civil, 2010. Rio de Janeiro: IBGE.

Jaramillo, J. M., Pérez, L., \& González, K. A. (2013). Metas de socialización maternas: Relación com edad, formación académica y zona habitacional. Revista Latinoamericana de Ciencias Sociales, Niñez y Juventud, 11(2), 719-739.

Jensen, T. M., \& Shafer, K. (2013). Stepfamily functioning and closeness: Children's view on second marriages and stepfather relationships. Social Work, 58(2), 127-136.

Kağitçibaşi, Ç. (1996). The autonomous-relational self: A new synthesis. European Psychologist, 1(3), 180-186.

Kağitçibaşi, Ç. (2005). Autonomy and relatedness in cultural context: Implications for self and family. Journal of Cross-Cultural Psychology, 36, 403-422.

Kağitçibaşi, Ç. (2007). Family, self, and human development across cultures: Theory and applications ( $2^{\text {nd }}$ ed.). Mahwah: Lawrence Erlbaum.

Kağitçibaşi, Ç. (2012). Socio-cultural change and integrative syntheses in human development: Autonomous-related self and social-cognitive competence. Child Development Perspectives, $6(1), 5-11$.

Kaslow, F. W. (2002). Families and family psychology at the millenium. American Psychologist, 56(1), 37-46.

Keller, H. (2012). Autonomy and Relatedness Revisited: Cultural manifestations of universal human needs. Child Development Perspectives, 6(1), 12-18.

Keller, H., \& Greenfield, P. M. (2000). History and Future of development in cross-cultural psychology. Journal of CrossCultural Psychology, 31(1), 52-62.

Keller, H., Borke, J., Yovsi, R., Lohaus, A., \& Jensen, H. (2005). Cultural orientations and historical changes as predictors of parenting behavior. International Journal of Behavioral Development, 29(3), 229-237. 
Keller, H., Lamm, B., Abels, M., Yovsi, R., Borke, J., Jensen, H., . . . Chaudhary, N. (2006). Cultural models, socialization goals, and parenting ethnotheories: A multi-cultural analysis. Journal of Cross-Cultural Psychology, 37(2), 155-172.

Leme, V. B. R., Del Prette, Z. A. P., \& Coimbra, S. (2013). Práticas Educativas Parentais e Habilidades Sociais de Adolescentes de Diferentes Configurações Familiares. Psico, 44(4), 560-570.

Ligthfoot, C. \& Valsiner J. (1992). Parental belief systems under the influence: social guidance of the construction of personal cultures. In I. S. Siegel, A. V. McGillicudy-DeLisi, \& J. Goodnow (Eds.), Parental belief systems: the psychological consequences for children (pp. 393-414). Hillsdale, NJ, Hove, UK: Lawrence Erlbaum.

Lins, Z. M. B., Salomão, N. M. R., Borges, L. C., Lins, S. L. B, \& Carneiro, T. F. (2015). Metas parentais de socialização em relação ao desenvolvimento de seus filhos. Interação em Psicologia, 19(1), 85-96.

Mendes, D. M. L. F., \& Pessôa, L. F. (2013). Comunicação afetiva nos cuidados parentais. Psicologia em Estudo, 18(1), 15-25.

Mota, C. P., \& Matos, P. M. (2011). Adolescência e conflitos parentais: uma perspectiva de resiliência. In P. M. Matos, C. Duarte \& M. E. Costa (Orgs.), Famílias: questões de desenvolvimento e intervenção (pp. 125-150). Porto: LivPsic.

Passos, M. C. (2015). Vicissitudes do tempo na formação dos laços familiares. In T. Féres-Carneiro (Org.), Família e Casal: Parentalidade e filiação em diferentes contextos (pp.11-23). Rio de Janeiro: Ed. PUC-Rio/Prospectiva.

Pessôa, L. F., Seidl-de-Moura, M. L., Ramos, D. O., \& Mendes, D. M. L. F. (2016). Sistemas de cuidados e o discurso de diferentes cuidadores do Rio de Janeiro: Evidências de trajetória de desenvolvimento. Estudos de Psicologia (Campinas), 33(1), 7182.

Pessôa, L. F., Ramos, D. O., Seidl-de-Moura, M. L., \& Silva, L. O. (2018). A valorização da obediência infantil como meta de socialização em diferentes configurações familiares. Psicologia Clínica, 30(1), 147-163.

Pinheiro, M. H. C., \& Biasoli-Alves, Z. M. M. (2008). A família como base. In L. Weber (Org.), Família e Desenvolvimento: Visões interdisciplinares. Curitiba: Juruá.

Seidl-de-Moura, M. L. (2003). Metas de socialização em mães primíparas em um modelo de cognições e valores parentais. Projeto de pesquisa não publicado, Rio de Janeiro: UERJ.

Seidl-de-Moura, M. L., Lordelo, E., Vieira, M. L., Piccinini, C. A., Siqueira, J. O., Magalhães, C. M. C., . . . Rimoli, A. (2008). Brazilian mothers socialization goals: Intracultural differences in 
seven Brazilian cities. International Journal of Behavioral Development, 32(6), 480-487.

Seidl-de Moura, M. L., Mendes, D. M. L. F, Mauro, M. L., Kobarg, A. P., Pessôa, L. F., \& Bandeira, T. T. A. (2013). Brazilian mothers' description of the children: Dimensions of autonomy and relatedness. Psicologia: Teoria e Pesquisa, 29(3), 249-255.

Seidl-de-Moura, M. L., Ramos, D. O., Pessôa, L. F., Carvalho, R. V. V., Victor, T. A. S., \& Mendes, D. M. L. F. (2017). Autonomiarelacionada como tendência do desenvolvimento do self: Novas evidências em um contexto brasileiro. Psicologia: Teoria e Pesquisa, 33, e3333, 1-9. doi: doi.org/10.1590/0102.3772e3333

Vieira, M. L., Martins, G. D. F., \& Lordelo, E. R. (2013). Independence and interdependence: The diversity of the Brazilian family from a culture perspective. Revista Interamericana de Psicología, $47(1), 111-120$.

Wagner, A. (2002). Família em cena. Tramas, dramas e transformações. Petrópolis: Vozes.

Westphal, J. P., Vieira, V., Vieira, M. L., \& Prado, A. B. (2010). O que mães pensam sobre seus filhos em três regiões distintas do Estado de Santa Catarina. Psicologia Argumento, Curitiba, 28(62), 235-246.

\section{Endereço para correspondência \\ Leticia Oliveira da Silva}

Pontifícia Universidade Católica do Rio de Janeiro - PUC-Rio

Programa de Pós-Graduação em Psicologia Clínica

Rua Marquês de São Vicente, 225, Gávea, CEP 22451-900, Rio de Janeiro - RJ, Brasil

Endereço eletrônico: leticia-oliveira92@hotmail.com

\section{Luciana Fontes Pessôa}

Pontifícia Universidade Católica do Rio de Janeiro - PUC-Rio

Programa de Pós-Graduação em Psicologia Clínica

Rua Marquês de São Vicente, 225, Gávea, CEP 22451-900, Rio de Janeiro - RJ, Brasil

Endereço eletrônico: pessoalf@gmail.com

Recebido em: 03/10/2018

Reformulado em: 29/12/2018

Aceito em: 07/01/2019

\section{Notas}

* Doutoranda do Programa de Pós-Graduação em Psicologia Clínica.

** Professora Adjunta do Programa de Pós-Graduação em Psicologia Clínica e Coordenadora do Departamento de Psicologia.

O presente artigo é um dos frutos da pesquisa de mestrado da primeira autora, na qual contei com a que obteve bolsa auxílio da CAPES. 
Este artigo de revista Estudos e Pesquisas em Psicologia é licenciado sob uma Licença Creative Commons Atribuição-Não Comercial 3.0 Não Adaptada. 